\title{
Proinflammatory Arterial Stiffness Syndrome: A Signature of Large Arterial Aging
}

\author{
Mingyi Wang Robert E. Monticone Kimberly R. McGraw \\ Laboratory of Cardiovascular Science, National Institute on Aging, National Institutes of Health, \\ Biomedical Research Center (BRC), Baltimore, MD, USA
}

\section{Keywords}

Aging · Chronic stress · Proinflammation · Arterial

remodeling $\cdot$ Arterial stiffening

\section{Abstract}

Age-associated structural and functional remodeling of the arterial wall produces a productive environment for the initiation and progression of hypertension and atherosclerosis. Chronic aging stress induces low-grade proinflammatory signaling and causes cellular proinflammation in arterial walls, which triggers the structural phenotypic shifts characterized by endothelial dysfunction, diffuse intimal-medial thickening, and arterial stiffening. Microscopically, aged arteries exhibit an increase in arterial cell senescence, proliferation, invasion, matrix deposition, elastin fragmentation, calcification, and amyloidosis. These characteristic cellular and matrix alterations not only develop with aging but can also be induced in young animals under experimental proinflammatory stimulation. Interestingly, these changes can also be attenuated in old animals by reducing low-grade inflammatory signaling. Thus, mitigating age-associated pro-

\section{KARGER}

Published by S. Karger AG, Basel inflammation and arterial phenotype shifts is a potential approach to retard arterial aging and prevent the epidemic of hypertension and atherosclerosis in the elderly.

This is a work of the U.S. Government and is not subject to copyright protection in the USA. Foreign copyrights may apply. Published by S. Karger AG, Basel

\section{Introduction}

Aging is a major risk factor for the morbidity and mortality caused by cardiovascular disease. Systemic aging is defined as an age-related decline in physiological function primarily driven by chronic exposure to low levels of sterile inflammation, known as "proinflammation," contributing to cellular senescence and pathological aging [1, 2]. Arterial aging is the cornerstone of systemic aging and is mainly driven by local proinflammation [3-5]. Ageassociated proinflammatory cellular and matrix modifications are the foundation for an exponential increase in the pathogenesis of hypertension and atherosclerosis $[3,6]$. 


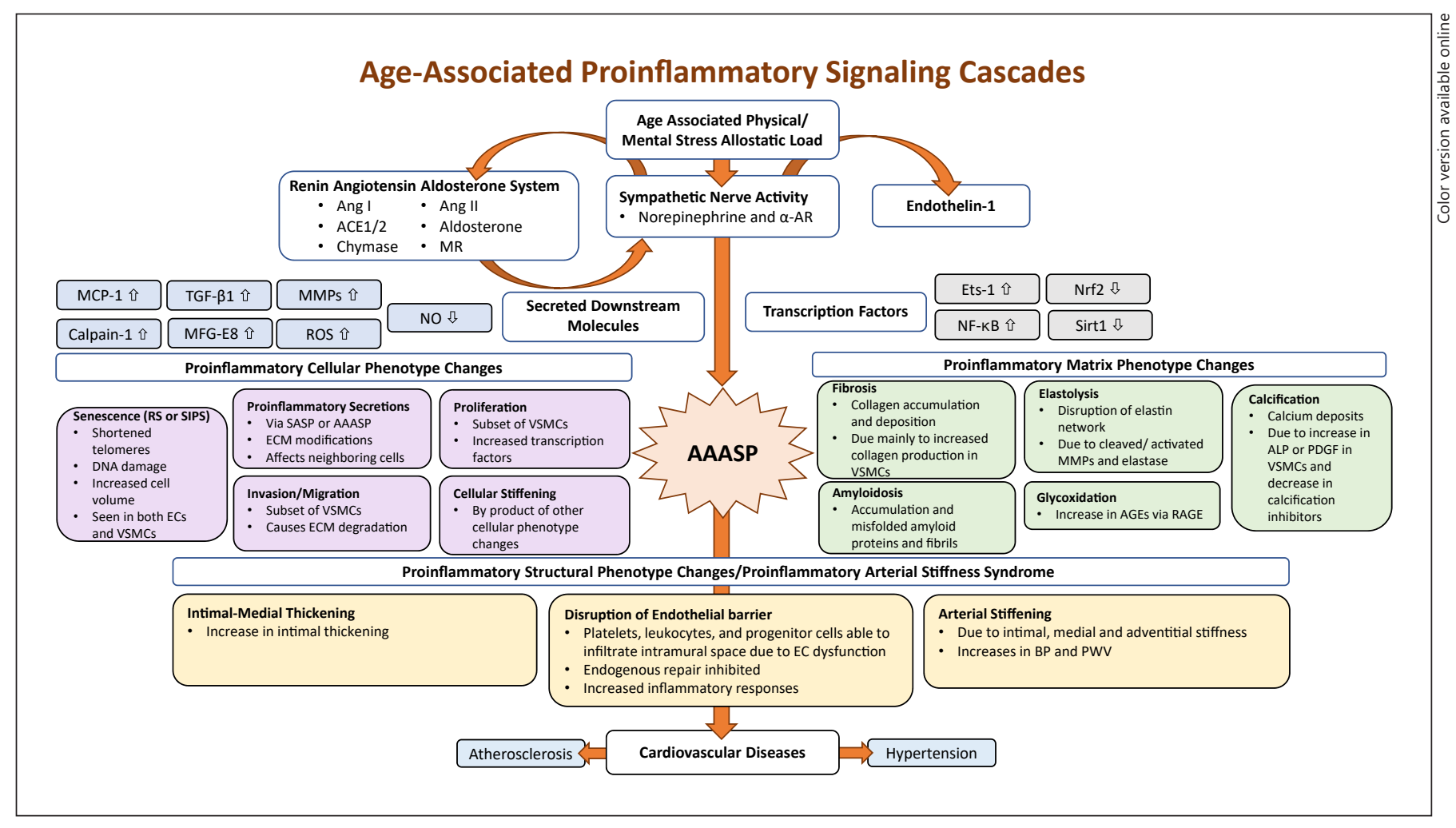

Fig. 1. Age-associated proinflammatory signaling cascades at the molecular, cellular, and tissue levels in the arterial wall. The ageassociated vascular molecular cascades are triggered by changes due to physical/mental stress allostatic load on the organism. These molecular changes impact the interconnected RAAS and the SNA systems, thus, activating ET-1, and lead to the secretion of downstream molecules and transcription factors. $\alpha-A R$, a-adrenergic receptor; ACE, angiotensin-converting enzyme; AAASP, age-associated arterial secretory phenotype; AGEs, advanced glycation end products; ALP, alkaline phosphatase; Ang, angiotensin; BP, blood pressure; EC, endothelial cell; ECM, extracellular matrix; Ets-1, the v-ets erythroblastosis virus E26 onco-

Age-associated arterial proinflammation is mainly generated by vascular endothelial cells (ECs) and vascular smooth muscle cells (VSMCs) [3]. Cellular proinflammation is tightly regulated by sympathetic nerve activity (SNA), the renin angiotensin aldosterone system (RAAS), and endothelin activities induced by physical and mental stress known as allostatic load [7-16]. This chronic stress is the cost that the organism pays for trying to maintain stability in a changing environment over a lifetime.

A comprehensive view of arterial aging is illustrated in Figure 1. At the molecular level, proinflammatory cytokines and chemokines accumulate within the arterial wall. At the cellular level, vascular cells shift phenotypically to heterogeneous phenotypes: a subset of VSMCs gene homolog 1; MCP-1, monocyte chemoattractant protein-1; MFG-E8, milk fat globule epidermal growth factor-8; MMPs, matrix metalloproteases; MR, mineralocorticoid receptor; NF- $\mathrm{kB}$, nuclear factor kappa-light-chain-enhancer of activated B cells; Nrf2, NF-E2-related factor 2; NO, nitric oxide; PDGF, plateletderived growth factor; PWV, pulse wave velocity; RAGE, receptor for advanced glycation end products; ROS, reactive oxygen species; RS, replicative senescence; SASP, senescence-associated secretory phenotype; Sirt1, silent information regulation 2 homolog 1 ; SIPS, stress-induced premature senescence; TGF- $\beta_{1}$, transforming growth factor- $\beta_{1}$; VSMC, vascular smooth muscle cell.

becomes senescent, while another subset of cells becomes more proliferative, invasive/migratory, secretory, and stiff. The extracellular matrix demonstrates fibrosis, elastolysis, calcification, amyloidosis, and glycoxidation. Finally, at the tissue level, proinflammation increases arterial intimal-medial thickening (IMT), endothelial dysfunction, arterial stiffening, and elevated blood pressure (BP). These tissue-level changes comprise "proinflammatory arterial stiffness syndrome," a clinical change that does not necessarily evolve into cardiovascular disease. Thus, inhibiting age-associated proinflammation may be a novel approach for maintaining a healthy vasculature and curbing the epidemic of cardiovascular disease in the aging population. 


\section{Molecular Phenotypes in the Aging Arterial Wall}

\section{Age-Associated Leading Stressors}

We recognize that increases in the key molecules of SNA, the RAAS, and endothelin-1 (ET-1) activity are the leading proinflammatory stressors in arterial aging based upon recently published studies (Fig. 1).

\section{Norepinephrine}

Aging increases SNA, which is characterized by an increase in neurohormone norepinephrine secretion in the arterial wall and an upregulation of its receptor, $\alpha$-adrenergic receptor $[9,10,17,18]$. SNA is also interconnected with the RAAS and ET-1. Increased SNA contributes to endothelial dysfunction, vasoconstriction, IMT, and BP increase and increases arterial proinflammation $[8,9,14,19]$.

\section{Angiotensin II}

Increased SNA triggers activation of the RAAS at both central and peripheral levels with aging $[9,12,14,19]$. The transcription, translation, and activity of angiotensin-converting enzyme (ACE) markedly increases in the arterial wall with aging [20-26]. Chymase, an alternative angiotensin convertase, also increases in expression within the arterial adventitia [22]. Both ACE and chymase cleave angiotensin I (Ang I) into angiotensin II (Ang II). Consequently, Ang II protein is significantly elevated in the aging arterial wall [21-23, 25, 27-29], along with increased expression of the Ang II receptor, AT1 [21, 30]. The "aging-elevated" Ang II/AT1 expression in the arterial wall signals to the SNA, subsequently creating an inflammatory environment which contributes to arterial remodeling.

\section{Aldosterone}

Aldosterone (Aldo), a component of the RAAS and a downstream Ang II effector, is also regulated by SNA, and is known as the sympathetic-adrenal system [9]. Aldo, secreted by the adrenal glands, binds to the mineralocorticoid receptors (MRs). Aging increases the clustered zona glomerulosa cells of the adrenal glands and subsequently enhances the production and secretion of Aldo, called "age-related autonomous aldosteronism" [31, 32]. Further, aging also increases the local expression of MR in arterial walls and cells [33-35]. Elevated Aldo/MR signaling enhances extracellular signal-regulated kinase (ERK) $1 / 2$ signaling, contributing to the proinflammatory phenotypic shift of VSMCs, thus promoting vasoconstriction, stiffening, and BP increase [33-37].

\section{Endothelin-1}

The endothelium, known as the body's largest "endocrine gland," produces ET-1, and is also regulated by SNA $[14,38]$. With advancing age, aortic proendothelin-1 (pro-ET-1) and activated matrix metalloproteinase (MMP) type II (MMP-2) levels increase [39]. Pro-ET-1 can be cleaved into an active ET-1 peptide by either endothelin-converting enzyme or MMP-2 within the arterial walls [39-41]. Consequently, active ET-1 levels are increased in aging $[38,39,42]$. ET-1 enhances inflammation by increasing the expression of the transcription factor E26 transformation-specific proto-oncogene 1 (ets-1) in VSMCs with aging [39]. Aging increases the sensitivity of the ET receptor in aortic walls, further augmenting proinflammation [43].

\section{Age-Associated Key Secreted Downstream Molecules}

The interrelationship of the SNA/RAAS/ET-1 signaling pathways promotes a proinflammatory response in aged arterial walls, leading to an increase in key downstream molecules (Fig. 1). Aged cells, including senescent cells, modify their microenvironment via the secretion of a variety of bioactive factors known as the age-associated arterial secretory phenotype (AAASP), including the senescence-associated secretory phenotype (SASP) [3].

\section{Monocyte Chemoattractant Protein-1}

Monocyte chemoattractant protein-1 (MCP-1) increases in aged arterial walls in association with both SNA and Ang II signaling [44, 45]. ET-1 also promotes the secretion of MCP-1 in aged VSMCs [39]. Both mRNA and protein levels of MCP-1 are upregulated within the aged aortic wall [45-47]. Increases in MCP-1 protein expression are mainly localized to the thickened intima and promote VSMC proinflammation [45-47]. MCP-1 is a key intermediary signaling molecule which connects the SNA/RAAS/ET-1 signaling pathway to proinflammatory cellular and matrix phenotypical changes.

\section{Transforming Growth Factor- $\beta_{1}$}

Transforming growth factor- $\beta_{1}$ (TGF- $\beta 1$ )/TGF beta receptor type II activation is a powerful fibrotic signaling cascade that is closely mediated by Ang II/AT1 signaling [4851]. The levels of secreted TGF- $\beta_{1}$ protein from VSMCs are upregulated in aged rat aortae [49]. Increased collagen synthesis, secretion, and deposition are triggered by interactions between TGF- $\beta_{1} /$ TGF beta receptor type II and pSMAD- $2 / 3$ signaling $[39,48,49,52]$. In addition, treating ECs with TGF- $\beta_{1}$ peptide also increases the expression of collagen types I and III $[53,54]$. Interestingly, MCP-1 has 
been shown to colocalize with TGF- $\beta_{1}$ within the arterial wall to enhance the activity of TGF- $\beta_{1}$ in VSMCs [55]. Thus, interactions of TGF- $\beta_{1}$ with MCP-1 may play a significant role in age-associated arterial fibrosis.

\section{Matrix Metalloproteinases}

MMPs degrade the extracellular matrix. MMP-2 is a downstream molecule of both Ang II and phenylephrine signaling in the arterial wall and cultured VSMCs [27]. Both the mRNA and protein levels of MMP-2/9 are upregulated in aged aortic walls [22, 27, 49,55-57]. The increased ratio of the MMP activator membrane-type 1 matrix metalloproteinase to the MMP tissue inhibitor of MMP-2 potentially promotes MMP-2/9 activation with aging [21, 22, 58]. Activated MMP-2/9 is predominantly located at the thickened intima and the inner media of arteries [22, 58]. Notably, secreted activated MMP-2/9 from VSMCs is upregulated with aging, which mainly contributes to an increased activation of arterial MMP-2/9. Importantly, activated MMP-2 increases the bioavailability of proinflammatory vasoactive molecules, e.g., cleaves latent transforming protein-1 and pro-ET- 1 into activated TGF- $\beta_{1}$ and ET- 1 in the arterial wall or VSMCs $[39-41,49]$.

\section{Calpain-1}

Calpain-1 is a calcium-dependent intracellular protease which modulates extracellular MMP- 2 and TGF- $\beta_{1}$ activity in the aged arterial wall or cultured VSMCs [29, 59]. The activity of calpain- 1 is significantly increased in both aged rat aortae and cultured aortic VSMCs [29] and facilitates activation of MMP-2 and TGF- $\beta_{1}$, leading to fibrosis and calcification [59]. In contrast, the calpain-1 inhibitor BDA-1 attenuates aortic calcification in aging klotho-deficient mice [60]. Ang II both activates and colocalizes with calpain-1 in the aged arterial wall and VSMCs [29]. Thus, calpain partially relays the proinflammatory signaling of Ang II in the aged arterial wall or cultured VSMCs.

\section{Milk Fat Globule-EGF-8}

Milk fat globule-EGF-8 (MFG-E8) is a highly glycosylated protein enriched in milk fat globule-containing EGF and blood-clotting factor VIII. Aging not only increases MFG-E8 mRNA and protein levels but also increases its fragment medin in the arterial wall [61-63]. Treating aged VSMCs with MFG-E8 increases the proliferation and migration of VSMCs. Medin has a strong affinity to elastin fibers, promoting elastolysis and amyloidosis in the arterial wall [62-65]. In addition, Ang II induces MFG-E8 protein expression, which increases MCP-1 ex- pression in VSMCs, promoting proinflammation [28]. Notably, MFG-E8 is also a well-known bridging molecule that mediates the clearance of apoptotic cells (efferocytosis) by macrophages, retarding the growth and vulnerability of atherosclerotic plaques in aging mice [66-68]. The complex role of MFG-E8 in the aged proinflamed arterial wall needs to be further explored.

\section{Reactive Oxygen Species}

Reactive oxygen species (ROS) are increased in the aged arterial wall or VSMCs. Nicotinamide adenine dinucleotide phosphate (NADPH) oxidase expression is the main source of production of arterial ROS. Further, levels of the antioxidant proteins $\mathrm{Cu} / \mathrm{Zn}$ SOD (SOD1), $\mathrm{Mg}$ SOD (SOD2), and extracellular matrix superoxide dismutase (ECM-SOD/SOD3) are downregulated during aging [69-72]. Therefore, aging creates an imbalance of NADPH oxidase and dismutase in the arterial wall, eventually augmenting the increase in ROS levels. This imbalance, along with increases in both Ang II and ET-1, enhances NADPH expression and the production of ROS $[14,25,52,69,73-77]$. Increased ROS modifies proinflammation, endothelial dysfunction, and arterial stiffening in the arterial wall with aging [25, 52, 72, 78-82].

\section{Nitric Oxide and Bioavailability}

Nitric oxide (NO), a small diffused signaling molecule, regulates arterial dilatation, stiffening, and inflammation with aging [25, 69, 72, 74, 83-87]. Endothelial NO synthase activation determines the production of $\mathrm{NO}$ in the arterial wall. Expression of arterial endothelial NO synthase is decreased and contributes to a reduction in $\mathrm{NO}$ production in the aged arterial wall [70, 84, 88-90]. In addition, NO interacts with ROS to generate peroxynitrite $\left(\mathrm{ONOO}^{-}\right)$. This ROS further decreases the bioavailability of NO, impairs endothelium-dependent relaxation, and enhances vasoconstriction and proinflammation $[74,90,91]$.

\section{Age-Associated Transcription Factors}

RAAS/SNA/ET-1 signaling contributes to the activation or inactivation of nuclear transcription factors that are key intermediary molecules contributing to proinflammatory cellular and matrix phenotypical changes (Fig. 1).

\section{Ets- 1 and NF- $\kappa \mathrm{B}$}

The major proinflammatory transcription factors Ets1 and NF- $\kappa \mathrm{B}$ are activated in the aged arterial wall. Increased Ets- 1 activity is closely associated with increased 
transcription levels of ET-1, MCP-1, TGF- $\beta_{1}$, and MMP2 [39]. Increased NF- $\kappa B$ activity in old arterial cells promotes oxidative stress and triggers an inflammatory response [92-94].

\section{Nrf2 and SIRT1}

The major anti-proinflammatory transcription factors nuclear factor (erythroid-derived 2)-like 2 (Nrf2) and sirtuin (silent mating type information regulation 2 homolog) 1 (S. cerevisiae) (SIRT1) are decreased in the aged arterial wall. Nrf2 protects against oxidative stress and its related cytotoxic effects and magnifies NF- $\kappa B$ activation [95]. SIRT1 is downregulated and inactivated with aging [71, 83, 87, 96, 97]. Decreased Sirt1 activity increases NADPH oxidase-dependent ROS production, senescence, and inflammation and enhances endothelial dysfunction in the aged arterial wall [83, 96-98].

\section{Summary}

With advancing age, both physical and mental stress increases due to continuous adaptations to changes in the living environment. Increased stress triggers the activation of both the RAAS and SNA, leading to ET-1 activation. These "leading proinflammatory signaling" events act on arterial cells by directly promoting the secretion or production of MCP-1, TGF- $\beta_{1}$, MMPs, calpain-1, and MFG-E8, known as the AAASP, as well as the activation or inactivation of transcription factors Ets-1, NF- $\kappa \mathrm{B}$, Nrf2, or Sirt1. These age-associated proinflammatory molecular phenotype alterations eventually lead to ageassociated cellular and matrix phenotypical changes. Further studies are needed to elucidate the finer details of the mutable events that facilitate the directionality and movement of the proinflammatory signaling cascade with aging as illustrated in Figure 1.

\section{Cellular and Matrix Phenotypes in the Aging Arterial Wall}

The structure and function of the arterial cells and matrix are remodeled with advancing age through proinflammatory signaling. Changes in age-associated cellular and extracellular phenotypes are illustrated in Figure 1.

\section{Age-Associated Cellular Phenotypes}

EC Senescence

The number of arterial ECs decreases with aging potentially due to either replicative senescence (RS) with telomere reduction and inactivation of telomerase, or stress-induced premature senescence (SIPS) without telomere involvement. Indeed, the number of both RS and SIPS ECs increases in the aged arterial wall [81, 85, $88,99-102]$. A subset of aged ECs appears with decreased mitotic frequency, an increase in cellular volume, and shortened telomeres while entering an RS state [103105]. In addition to RS, the Ang II signaling cascade plays a significant role in the SIPS of ECs via a reduction of Sirt1 and ERK1/2/BCL2 signaling, and functional autophagy in addition to increased ROS production [81, 100-102].

\section{VSMC Senescence}

A subset of aged VSMCs appears enlarged and becomes senescent in the arterial wall $[97,98,100,106-$ 110]. Oxidative stress and DNA damage cause VSMC senescence and are linked to shortened telomeres or SIPS triggered through Ang II signaling [82, 100, 111, 112]. Increased senescence is associated with an increase in p16 expression, a loss of SIRT1 expression, and an upregulation of miR-34a in VSMCs. In addition, the mutant lamin A, known as progerin, also drives the senescence of VSMCs $[110,113]$.

\section{Proliferation}

VSMC proliferation is increased in the aged arterial wall. Age-associated secretory molecules, such as MFGE8, may promote a replicative subset of VSMCs [108, 114]. This subset of VSMCs has an increased proliferative capacity via MFG-E8 signaling, displaying increased ERK1/2 phosphorylation and 5-bromo-2'-deoxyuridine incorporation, as well as increased expression of proliferating cellular nuclear antigen, cyclin-dependent kinase 4, and platelet-derived growth factor (PDGF-BB) [115].

\section{Invasion/Migration}

VSMC invasion is the ability to migrate or infiltrate neighboring tissue through the vascular extracellular matrix. Interestingly, the invasive capacity of a VSMC subset is increased in the aged arterial wall, contributing to diffuse intimal thickening $[3,108,114,116]$. Aging increases the signaling of Ang II, which triggers the secretion of calpain-1, MFG-E8, or MCP-1 in VSMCs, and also activates MMP-2 [27-29]. Increased MMP-2 activation is the key molecule that drives the invasive capabilities of VSMCs via a breakdown of their basement membrane and surrounding extracellular matrix [21, $28,29,55]$. Conversely, the invasive capacity of old VSMCs can be inhibited by the MMP-2 inhibitor GM6001 [46, 55]. 
Stiffening

Stiffening of VSMCs is a central and mutable element in arterial aging [78, 117-121]. VSMCs derived from older animals demonstrate increased stiffness compared to similar cells derived from young adults $[119,122]$. VSMC stiffness is highly sensitive to microenvironmental molecules such as TGF- $\beta_{1}$ and transglutaminase $2[119,123]$. TGF- $\beta_{1}$ serves as a specific modifier of age-associated VSMC stiffening through the clustering of mechanosensitive $\alpha_{5} \beta_{1}$ and $\alpha_{v} \beta_{3}$ integrins [122]. Increased stiffness in aging is also dependent on the expression and organization of the VSMC cytoskeletal proteins along the arterial tree $[123,124]$. Interestingly, a stiffened substrate reinforces VSMC stiffening [125]. The stiffness correlates with phenotypic changes of VSMCs [123]. Increased stiffening is converted into proinflammatory signaling in the aged arterial wall [126].

\section{Proinflammatory Secretions}

Aged vascular cells, including senescent cells, modify their microenvironment via the autonomous or nonautonomous secretion of a variety of bioactive factors. The vascular SASP includes cells that promote proinflammation of neighboring cells $[108,114]$. Interestingly, growing evidence indicates that aged primary isolated VSMCs from aortic walls have a unique chemokine and cytokine proinflammatory profile (the AAASP), which also drives proinflammation in neighboring cells $[5,92]$. MMP-2, MCP1 , TGF- $\beta_{1}$, MFG-E8, and TNF- $\alpha$ are characteristic proteins secreted from old VSMCs with the AAASP $[3,92]$.

\section{Age-Associated Matrix Phenotypical Changes}

The extracellular matrix of arterial walls is modified by age-associated proinflammatory secretions of vascular cells through fibrosis, elastolysis, calcification, and amyloidosis $[47,85,97,99,127]$.

\section{Fibrosis}

Fibrosis develops through an increase in collagen deposition in the arterial walls of aging rats $[58,75]$. Collagen accumulation also significantly increases within the arterial walls of aged humans [128]. Secreted MMP-2 activates TGF- $\beta_{1}$ and promotes VSMC collagen production $[108,114]$. Collagen deposition within the interlamellar layers of the arterial wall plays a significant mechanical role in arterial stiffening [129].

\section{Elastolysis}

An intact interlamellar elastin layer is important for the health of large arteries. The aging interlamellar elastin network is disrupted and collapsed in elastolysis due to cleavage by MMPs and elastase [58, 130-132]. Elastolysis is observed with an increase in the amounts of activated MMP-2/9 or elastase in the interlamellar elastin network $[50,55]$. This age-associated destruction of the interlamellar elastin lamina is also associated with loss of tropoelastin production, impairing rejuvenation [133]. The destruction of vascular interlamellar elastin results in an eventual decrease in arterial elastic energy storage capability, compliance, and resilience [134]. In addition, short peptides, released during elastolysis, known as "elastokines," actively participate in the onset and progression of arterial inflammation and calcification.

\section{Calcification}

Arterial calcification plays a crucial role in the development of arterial stiffening [135]. Increased calcium deposits, an element of calcification, are markedly increased in the arterial wall with aging $[136,137]$. The morphology of older VSMCs appears osteoblast-like, producing large amounts of bone-like substrates such as collagen II [59]. The development of arterial calcification is dependent upon a balance of pro- and anti-calcification molecules. Overexpression of alkaline phosphatase, a pro-calcification molecule, increases arterial calcification and is one of the pro-calcification molecules that is found with greater frequency in old or senescent VSMCs. In addition, anticalcification molecules, such as osteonectin and osteopontin, are simultaneously reduced in old VSMCs [59]. Notably, age-associated increases in PDGF, a powerful cellular mitogen, also significantly accelerate the process of arterial calcification [138].

\section{Amyloidosis}

With advancing age, misfolded aggregated amyloid proteins and fibrils are increased in arterial walls [62-65]. One of the main constituents of arterial amyloid fibrils is a 5.5-kDa fragment of MFG-E8, known as medin, which is markedly increased in the aged arterial wall $[61,62,65]$. Medin has a high capacity for binding to elastin fibers, potentially increasing stiffness and calcification [61-65]. Thus, medin amyloid is implicated as greatly affecting the elasticity of aged arteries and therefore needs further investigation.

\section{Advanced Glycation End Products}

Advanced glycation end products (AGEs) are increased in the aged arterial wall. It is well known that AGE accumulation contributes to multiple structural and functional alterations in the arterial system, such as 
senescence, proinflammation, and stiffening $[4,52,139$ 141]. AGEs are often generated by reactions between sugar chains and biologic amines of oxidized collagen. Older, cleaved/degraded, oxidized collagen fibers are common molecular targets that are easily modified via a reaction between ROS and sugars in the arterial wall. Aging increases AGEs and promotes collagen production through activation of its receptor, RAGE, in a feedback manner.

\section{Summary}

Aging can be described as a form of subclinical pathological conditions. Proinflammatory molecular signaling acts on arterial cells, generating age-associated cellular and matrix phenotypical changes, as illustrated in Figure 1. These phenotypes are all observed in the aged arterial wall and have been reproduced in vivo and in vitro. These characteristic cellular and matrix alterations can also be induced in young animals under experimental proinflammatory stimulation. Clinical proinflammatory structural phenotypical changes may be observed in aged populations without evidence of cardiovascular disease. The signaling by these phenotypes is complex, and it is unknown how multiple cellular and matrix events are controlled and how a subclinical symptom evolves into a clinical disease. For example, how does the arterial stiffness syndrome evolve into a clinical pathological condition such as hypertension and atherosclerosis? It is important to decode the signaling network and find the key switch for the diagnosis, prevention, and treatment of adverse arterial remodeling with aging.

\section{Arterial Subclinical Phenotypes with Aging}

Age-associated cellular and extracellular phenotypic shifts ultimately lead to "arterial proinflammatory stiffness syndrome," including IMT, endothelial dysfunction, stiffening, and BP increase (Fig. 2).

\section{Intimal-Medial Thickening}

IMT can be accurately evaluated by B-mode ultrasound and other noninvasive imaging techniques and is a hallmark of age-associated arterial remodeling $[3,8,23$, $116,142-149]$. Expansion of the intimal layer, rather than the media, is mainly responsible for increased IMT [150], which is linked to increases in both vascular relaxation and stiffening $[143,144,151,152]$. Notably, there is also an increase in VSMC progenitor cells promoting ageassociated IMT [153].

\section{Endothelial Dysfunction}

The arterial endothelial barrier becomes disrupted with advancing age. More senescent ECs, with reduced telomerase, contribute to endothelium-dependent dysfunction by increasing the number of defective sites along the lumen $[85,88,99,154,155]$. Circulating platelets adhere and infiltrate via the damaged sites into the arterial wall, initiating inflammatory responses. Increased adhesive platelets on the inner surface of the arterial lumen not only inhibit the proliferation and migration of local ECs but also exhaust endogenous repair by progenitor cells [99]. Therefore, increases in activated and aggregated platelets damage the integrity of the aged arterial endothelial barrier and also promote endothelial dysfunction [145]. Furthermore, EC contractility is enhanced with aging, leading to increased endothelial permeability and intimal stiffening $[25,26,38,72,83,85,87$, 93, 148].

Aging also increases monocytosis and enhances macrophage transdifferentiation and accumulation within the aorta $[145,156]$. The accumulation of macrophages within the arterial wall leads to metabolic impairment and subsequently accelerates arterial remodeling [145, 157]. Increased amounts of activated neutrophils and lymphocytes infiltrate the intramural space and interact with ECs and VSMCs, facilitating ROS production, senescence, and endothelial function [68, 145, 158, 159]. In addition, older subjects with greater cardiovascular risk factors have lower numbers of circulating endothelial progenitor cells, which is linked to the endogenous regenerative potential, suggesting the reparative capacity of the endothelium is decreased [142].

\section{Arterial Stiffening}

Arterial stiffness, including intimal, medial, and adventitial stiffness, is dependent on an intrinsic stress/ strain relationship determined by both cell and matrix stiffness [77, 118, 120, 123, 125, 135, 139, 147, 148, 151, $154,160-163]$. Pulse wave velocity (PWV) has emerged as a gold standard for the noninvasive assessment of central arterial stiffness, a predictor of the incidence of hypertension and all-cause mortality, and increases with advancing age [144, 151, 162-164]. The Baltimore Longitudinal Study of Aging (BLSA) demonstrated that increased PWV was associated with increased systolic BP and also a greater incidence of hypertension [165]. Further, the aortic-brachial PWV ratio has emerged as a novel index of BP independent of vascular aging [144], and the carotid-radial/carotid-femoral PWV ratio is an accurate predictor of all-cause mortality [151]. 


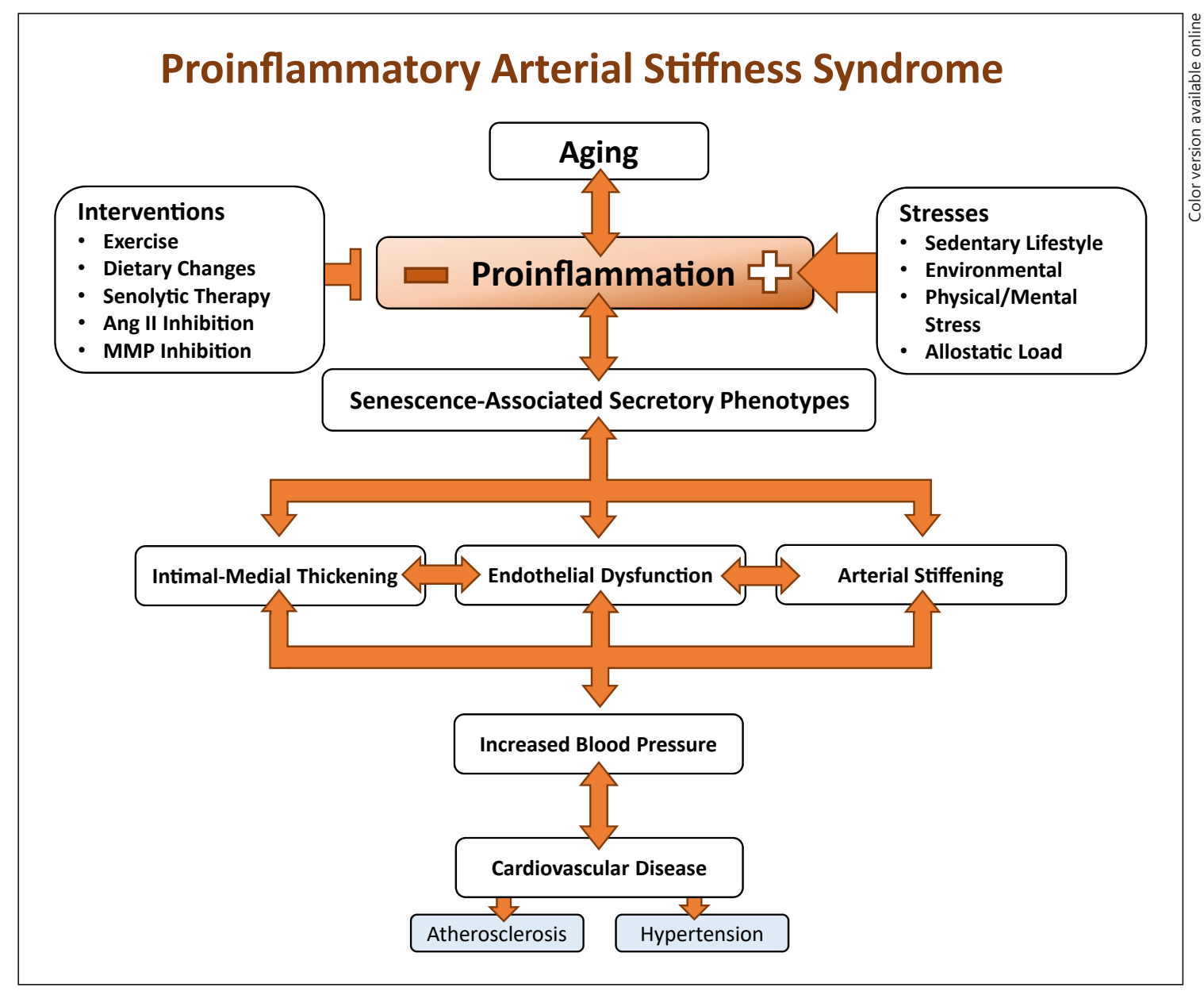

Fig. 2. Diagram of proinflammatory arterial stiffness syndrome. The phenotype changes made at the cellular and matrix level characterize the final stage of the vascular aging cascade that ultimately leads to proinflammatory structural phenotype changes and eventually cardiovascular disease. Ang II, angiotensin II; MMP, matrix metalloprotease.

\section{BP Increase}

Increases in systolic BP and pulse pressure occur after the sixth decade of life, becoming a hallmark of arterial aging $[135,143-145,147,160,166]$. Further, BP measurements are closely associated with PWV [143].

\section{Summary}

The accumulation of the above-mentioned molecular, cellular, and matrix phenotypes in the arterial wall with aging manifests as arterial proinflammatory stiffness syndrome. The clinical arterial phenotype is illustrated in Figure 2, including IMT, endothelial dysfunction, stiffening, and BP increase. These subclinical conditions are detected in the elderly without overt cardiovascular events, which we regard as arterial proinflammatory syndrome.
Further studies are needed to understand the mechanism by which accelerated aging leads to the clinical arterial phenotype and reduces the disease threshold which may ultimately lead to clinical cardiovascular disease.

\section{Interventions to Counteract Arterial Aging}

Since proinflammation is central to arterial aging, efforts to reduce proinflammation could help reduce the clinical progression of arterial aging. A healthy lifestyle and regular exercise can prevent age-associated senescence and secretion.

Pharmacological interventions may act to disrupt the progression of vascular aging by inhibiting the AAASP/ 


\section{Proinflammatory Stiffness Syndrome Interventions}

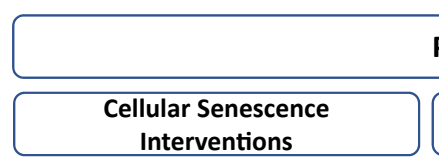

Pharmacological Interventions

\begin{tabular}{|l|}
\hline Senolytic therapy \\
- \\
Selectively induces death of \\
- Improvent cells \\
bioavailability \\
- Improves vasomotor function \\
- E.g. dasatinib and quercetin
\end{tabular}

Aging-Associated Secretion
Interventions

Vascular Aging Interventions

Ang II Inhibition
- Reduces proinflammatory molecules

- Retards aortic remodeling progression and endothelial dysfunction

- E.g. $\beta$-adrenergic blockers, ACE inhibitors, AT1 blockers

MMP Inhibition

- Retards increases in gelatinase and interstitial collagenase activity

- Retards increases in BP

- E.g. Statin therapies

\section{SIRT1 Activation}

- Resveratrol Treatment

- Retards vascular senescence and proinflammatory molecules

- Mimics Caloric Restriction

mTOR inhibitor

- Rapamycin Treatment

- Decreases inflammation and hypertrophy

- Metformin Treatment

- Increases in blood flow reduction in systolic $\mathrm{BP}$ and vasodilation improvements

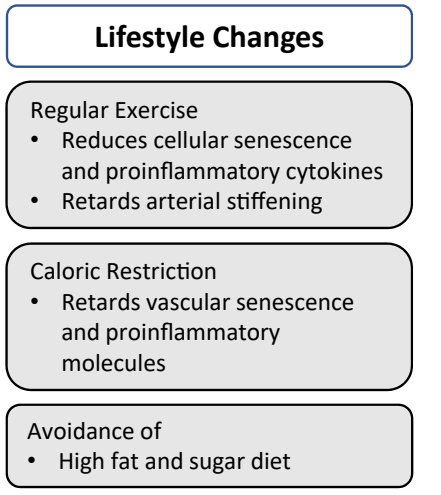

Retardation or Prevention of Arterial Aging

Decrease susceptibility to proinflammatory risk factors

- Increase threshold of insults by risk factors

- Curb the epidemic of quintessential cardiovascular disease

Fig. 3. Age-associated proinflammatory arterial stiffness syndrome interventions. Vascular aging can be mitigated through various approaches that target the proinflammatory cascades. The eventual structural phenotypical changes are not inevitable, and cardiovascular disease may be prevented. ACE, angiotensin-converting enzyme; Ang II, angiotensin II; AT1, Ang II receptor; BP, blood pressure; MMP, matrix metalloprotease; mTOR, mammalian target of rapamycin; SIRT1, silent information regulation 2 homolog 1 .

SASP directly or, through the removal of senescent vascular cells with senolytic drugs, may modify arterial aging and diminish the proinflammatory cascades [2, 167-169] (Fig. 3).

\section{Diet}

Long-term caloric restriction retards both vascular senescence and proinflammatory molecules by decreasing MCP-1, ROS, and MMP activation and improving NO bioavailability and endothelial function, thereby preventing arterial stiffening with aging $[86,170-172]$. Not only are these molecules disrupted, but the clinical signs of aging - such as increased body weight, BP, cholesterol levels, and arterial stiffness - improve [86, 170-172].

A high-fat-and-sugar (HFS) diet drives arterial aging in nonhuman primates [170,171]. Animals fed on an HFS diet for 2 years not only showed increases in body weight and circulating cholesterol, but also exhibited signs of central arterial wall stiffening and inflammation [164]. Further study showed that the stiffening-associated loss of EC integrity, lipid and macrophage infiltration, and calcification of the arterial wall were driven by genomic and proteomic disorders of oxidative stress and inflammation [164].

\section{Exercise}

Regular exercise substantially reduces ET-1 signaling, TGF- $\beta_{1}$ activity, cellular senescence, proinflammation, and calcification in the aged arterial wall $[76,78,86,89$, $141,173]$. Importantly, regular exercise effectively prevents age-associated SNA, BP increase, arterial stiffening, and endothelial dysfunction $[11,16,78,89,152,174]$.

\section{Senolysis}

Clearance of senescent cells using transgenic and pharmacological approaches retards arterial aging. Senolytics, such as dasatinib and quercetin, have been utilized to retard vascular aging. Dasatinib primarily eliminates senescent progenitor cells, while quercetin is more effective 
against senescent ECs [175]. Combined treatment with dasatinib and quercetin significantly reduces the arterial senescent cell burden in the arterial wall, increases NO bioavailability, and improves vasomotor dysfunction with aging [85]. Senolytic therapy can also reduce the progress of age-associated atherosclerosis $[176,177]$.

\section{Inhibition of Ang II Signaling}

The Ang II proinflammatory signaling cascade has been widely studied $[20,24-26,44,81,178]$. Chronic administration of Ang II to young rats not only increases their BP, but also enhances the activity of MMP-2, TGF- $\beta_{1}$, calpain-1, and MFG-E8 as well as collagen production within the arterial wall, similar to that in untreated old animals $[27,29,59$, $143,179]$. In contrast, chronic ACE inhibition of the Ang II receptor, an $\mathrm{AT}_{1}$ antagonist, significantly reduces the abundance of proinflammatory molecules and retards the progression of adverse aortic remodeling in experimental aging animals [20, 24-26, 178].

\section{Inhibition of MMPs}

Activated MMPs are common elements of the SASP and AAASP. Chronic administration of PD166793, a broad-spectrum MMP inhibitor, significantly retards age-associated increases in gelatinase and interstitial collagenase activity, ET-1 expression, elastic fiber fragmentation, and collagen production in the arterial wall of rats [39]. Interestingly, MMP inhibition also substantially retards increases in BP with advancing age [39].

\section{Activation of SIRT1}

Resveratrol treatment, a caloric restriction-mimicking small molecule and an agonist of SIRT1, effectively prevented HFS diet-induced arterial wall inflammation and arterial stiffening in nonhuman primates [164]. These findings suggest that dietary resveratrol, like caloric restriction, promises to ameliorate age-associated arterial inflammation, elastolysis, and stiffening $[81,92,96,180]$.

\section{Inhibition of mTOR}

Other treatments, like rapamycin and metformin therapy, focus on decreasing inflammation and arterial stiffening and improving endothelial dysfunction through the inhibition of mTOR (mammalian target of rapamycin) $[181,182]$.

\section{Summary}

Pharmacological and lifestyle interventions against ageassociated proinflammatory stiffness syndrome are illustrated in Figure 3. These interventions are largely derived from studies of experimental animals, and it is difficult to translate these approaches to the clinic. It is important to perform a large double-blind, random clinical trial to find the most effective time, dose, and side effects of potential treatments. Since advanced aging is a form of subclinical disease, targeting proinflammation may be the best approach to mitigating cardiovascular disease, which evolves into clinical conditions through either reduced disease thresholds or increased susceptibility and vulnerability.

\section{Concluding Remarks}

Age-associated arterial structural and functional remodeling are driven by chronic increases in proinflammatory signaling causing proinflammatory stiffness syndrome. At the cellular level, increases in senescent and senescenceassociated molecular signaling have been observed both in vivo and in vitro. Microscopically, IMT, endothelial disruption, cellular senescence, and senescence-associated cellular and matrix phenotypes are characteristics of the aged arterial wall. These adverse molecular, cellular, and matrix events, presenting as "old age phenotypes," are also observed in young animals experimentally infused with proinflammatory stimulants. Alternatively, in old animals, these adverse remodeling events are alleviated by inhibition of cellular senescence and senescence-associated phenotypes, resulting in more "youthful phenotypes."

Arterial senescence and senescence-associated heterogeneous phenotypes cause proinflammatory stiffness syndrome, presenting as a subclinical condition, and they may prepare the ground for the initiation and progression of hypertension and atherosclerosis at the molecular, cellular, and vascular levels. Thus, interventions that suppress or prevent proinflammatory stiffness syndrome at different levels may hold great promise in treating and preventing age-associated vascular diseases such as hypertension and atherosclerosis. Questions still remain regarding the mutability of the proinflammatory cascades and the triggers that control each level. Future studies are needed to decode the proinflammatory signaling network and understand how subclinical conditions evolve into cardiovascular diseases.

\section{Acknowledgements}

I, Mingyi Wang, would like to thank my advisor and mentor Edward G. Lakatta, MD, chief of the Laboratory of Cardiovascular Science, National Institution on Aging, National Institutes of Health, for introducing me to the wonders and frustrations of scientific research on cardiovascular aging. 


\section{Disclosure Statement}

The authors have no conflicts of interest to disclose.

\section{Funding Sources}

This research was supported by the Intramural Research Program of the National Institute on Aging, National Institutes of Health. The findings and conclusions of this report are those of the authors and do not necessarily represent the official position of the National Institute on Aging, National Institutes of Health.

\section{References}

1 Michaud M, et al: Proinflammatory cytokines, aging, and age-related diseases. J Am Med Dir Assoc 2013;14:877-882.

2 Soto-Gamez A, Demaria M: Therapeutic interventions for aging: the case of cellular senescence. Drug Discov Today 2017;22:786795.

3 Wang $M$, et al: Proinflammation: the key to arterial aging. Trends Endocrinol Metab 2014;25:72-79.

4 Wang M, Khazan B, Lakatta EG: Central arterial aging and angiotensin II signaling. Curr Hypertens Rev 2010;6:266-281.

5 Wang M, Monticone RE, Lakatta EG: Arterial aging: a journey into subclinical arterial disease. Curr Opin Nephrol Hypertens 2010;19: 201-207.

6 Go AS, et al: Heart disease and stroke statistics - 2014 update: a report from the American Heart Association. Circulation 2014; 129:e28-e292.

7 Wang M, Shah AM: Age-associated pro-inflammatory remodeling and functional phenotype in the heart and large arteries. J Mol Cell Cardiol 2015;83:101-111.

8 Dinenno FA, et al: Age-associated arterial wall thickening is related to elevations in sympathetic activity in healthy humans. Am J Physiol Heart Circ Physiol 2000;278:H1205H1210.

9 Gordon $\mathrm{RD}$, et al: Role of the sympathetic nervous system in regulating renin and aldosterone production in man. J Clin Invest 1967; 46:599-605

10 Kaplon RE, Walker AE, Seals DR: Plasma norepinephrine is an independent predictor of vascular endothelial function with aging in healthy women. J Appl Physiol (1985) 2011; 111:1416-1421.

11 Tanaka H, Dinenno FA, Seals DR: Reductions in central arterial compliance with age are related to sympathetic vasoconstrictor nerve activity in healthy men. Hypertens Res 2017;40: 493-495.

12 Arnold AC, Gallagher PE, Diz DI: Brain renin-angiotensin system in the nexus of hypertension and aging. Hypertens Res 2013;36:513.

13 Barnes JN, et al: Aging enhances autonomic support of blood pressure in women. Hypertension 2014;63:303-308.

14 Bruno RM, et al: Sympathetic regulation of vascular function in health and disease. Front Physiol 2012;3:284.
15 Kaye DM, Esler MD: Autonomic control of the aging heart. Neuromolecular Med 2008; 10:179-186.

16 Laitinen T, et al: Age dependency of cardiovascular autonomic responses to head-up tilt in healthy subjects. J Appl Physiol (1985) 2004; 96:2333-2340.

17 Barrett-O'Keefe Z, et al: Angiotensin II potentiates $\alpha$-adrenergic vasoconstriction in the elderly. Clin Sci (Lond) 2013;124:413-422.

18 Rudner XL, et al: Subtype specific regulation of human vascular $\alpha_{1}$-adrenergic receptors by vessel bed and age. Circulation 1999;100: 2336-2343.

$19 \mathrm{Xu} \mathrm{B}, \mathrm{Li} \mathrm{H}$ : Brain mechanisms of sympathetic activation in heart failure: roles of the reninangiotensin system, nitric oxide and proinflammatory cytokines (Review). Mol Med Rep 2015;12:7823-7829.

20 Michel JB, et al: Effect of chronic ANG I-converting enzyme inhibition on aging processes. II. Large arteries. Am J Physiol 1994;267(pt 2):R124-R135.

21 Wang M, et al: Proinflammatory profile within the grossly normal aged human aortic wall. Hypertension 2007;50:219-227.

22 Wang M, et al: Aging increases aortic MMP-2 activity and angiotensin II in nonhuman primates. Hypertension 2003;41:1308-1316.

23 Harvey A, Montezano AC, Touyz RM: Vascular biology of ageing - implications in hypertension. J Mol Cell Cardiol 2015;83:112-121.

24 Basso N, et al: Protective effect of long-term angiotensin II inhibition. Am J Physiol Heart Circ Physiol 2007;293:H1351-H1358.

25 Flavahan S, Chang F, Flavahan NA: Local renin-angiotensin system mediates endothelial dilator dysfunction in aging arteries. Am J Physiol Heart Circ Physiol 2016;311:H849H854.

26 Mukai Y, et al: Inhibition of renin-angiotensin system ameliorates endothelial dysfunction associated with aging in rats. Arterioscler Thromb Vasc Biol 2002;22:1445-1450.

27 Wang M, et al: Angiotensin II activates matrix metalloproteinase type II and mimics age-associated carotid arterial remodeling in young rats. Am J Pathol 2005;167:1429-1442.

$28 \mathrm{Fu} \mathrm{Z}$, et al: Milk fat globule protein epidermal growth factor-8: a pivotal relay element within the angiotensin II and monocyte chemoattractant protein-1 signaling cascade mediating vascular smooth muscle cells invasion. Circ Res 2009; 104:1337-1346.
29 Jiang L, et al: Increased aortic calpain-1 activity mediates age-associated angiotensin II signaling of vascular smooth muscle cells. PLoS One 2008; 3:e2231.

30 Nishimura A, et al: Purinergic $\mathrm{P}_{2} \mathrm{Y}_{6}$ receptors heterodimerize with angiotensin AT1 receptors to promote angiotensin II-induced hypertension. Sci Signal 2016;9:ra7.

31 Nanba K, et al: Age-related autonomous aldosteronism. Circulation 2017;136:347-355.

32 Seals DR, Esler MD: Human ageing and the sympathoadrenal system. J Physiol 2000; 528(pt 3):407-417.

33 Krug AW, et al: Elevated mineralocorticoid receptor activity in aged rat vascular smooth muscle cells promotes a proinflammatory phenotype via extracellular signal-regulated kinase $1 / 2$ mitogen-activated protein kinase and epidermal growth factor receptor-dependent pathways. Hypertension 2010;55:14761483.

34 DuPont JJ, et al: Vascular mineralocorticoid receptor regulates microRNA-155 to promote vasoconstriction and rising blood pressure with aging. JCI Insight 2016;1:e88942.

35 McCurley A, et al: Direct regulation of blood pressure by smooth muscle cell mineralocorticoid receptors. Nat Med 2012;18:14291433.

36 Galmiche G, et al: Smooth muscle cell mineralocorticoid receptors are mandatory for aldosterone-salt to induce vascular stiffness. Hypertension 2014;63:520-526.

37 Koenig JB, Jaffe IZ: Direct role for smooth muscle cell mineralocorticoid receptors in vascular remodeling: novel mechanisms and clinical implications. Curr Hypertens Rep 2014; 16:427.

38 Goel A, et al: Increased endothelial exocytosis and generation of endothelin- 1 contributes to constriction of aged arteries. Circ Res 2010; 107:242-251.

39 Wang M, et al: Chronic matrix metalloproteinase inhibition retards age-associated arterial proinflammation and increase in blood pressure. Hypertension 2012;60:459-466.

40 Abdalvand A, et al: Matrix metalloproteinase enhances big-endothelin-1 constriction in mesenteric vessels of pregnant rats with reduced uterine blood flow. Hypertension 2013; 61:488-493. 
41 Lekontseva ON, et al: Ovariectomy in aged versus young rats augments matrix metalloproteinase-mediated vasoconstriction in mesenteric arteries. Menopause 2010;17:516523.

42 Simeone SM, et al: Vascular gene expression in mice overexpressing human endothelin-1 targeted to the endothelium. Physiol Genomics 2011;43:148-160.

43 Tomobe Y, et al: Mechanisms of altered sensitivity to endothelin- 1 between aortic smooth muscles of spontaneously hypertensive and Wistar-Kyoto rats. J Pharmacol Exp Ther 1991;257:555-561.

44 Hao Q, et al: Curcumin attenuates angiotensin II-induced abdominal aortic aneurysm by inhibition of inflammatory response and ERK signaling pathways. Evid Based Complement Alternat Med 2014;2014:270930.

$45 \mathrm{Lu} \mathrm{XT}$, et al: Chronic psychological stress induces vascular inflammation in rabbits. Stress 2013;16:87-98.

46 Spinetti G, et al: Rat aortic MCP-1 and its receptor CCR2 increase with age and alter vascular smooth muscle cell function. Arterioscler Thromb Vasc Biol 2004;24:1397-1402.

47 Ferlosio A, et al: Age-related increase of stem marker expression influences vascular smooth muscle cell properties. Atherosclerosis 2012;224:51-57.

$48 \mathrm{Lu}$ P, et al: Role of TGF- $\beta 1 / \mathrm{Smad} 3$ signaling pathway in secretion of type I and III collagen by vascular smooth muscle cells of rats undergoing balloon injury. J Biomed Biotechnol 2012;2012:965953.

49 Wang M, et al: Matrix metalloproteinase 2 activation of transforming growth factor- $\beta 1$ (TGF- $\beta 1$ ) and TGF- $\beta 1$-type II receptor signaling within the aged arterial wall. Arterioscler Thromb Vasc Biol 2006;26:1503-1509.

50 Randell A, Daneshtalab N: Elastin microfibril interface-located protein 1, transforming growth factor beta, and implications on cardiovascular complications. J Am Soc Hypertens 2017;11:437-448.

51 Tang Y, et al: Mechanisms of TGF- $\beta$-induced differentiation in human vascular smooth muscle cells. J Vasc Res 2011;48:485-494.

52 Lozhkin A, et al: NADPH oxidase 4 regulates vascular inflammation in aging and atherosclerosis. J Mol Cell Cardiol 2017;102:10-21.

53 Chiasson VL, et al: Endothelial cell transforming growth factor- $\beta$ receptor activation causes tacrolimus-induced renal arteriolar hyalinosis. Kidney Int 2012;82:857-866.

54 Bai H, et al: Suppression of transforming growth factor- $\beta$ signaling delays cellular senescence and preserves the function of endothelial cells derived from human pluripotent stem cells. Stem Cells Transl Med 2017;6:589600.

55 Wang M, et al: A local proinflammatory signalling loop facilitates adverse age-associated arterial remodeling. PLoS One 2011;6:e16653.

$56 \mathrm{Li} \mathrm{Z}$, et al: Increased expression of matrix metalloproteinase- 2 in the thickened intima of aged rats. Hypertension 1999;33:116-123.
57 Spiers JP, et al: Alterations in vascular matrix metalloproteinase due to ageing and chronic hypertension: effects of endothelin receptor blockade. J Hypertens 2005;23: 1717-1724.

58 Wang M, Lakatta EG: Altered regulation of matrix metalloproteinase- 2 in aortic remodeling during aging. Hypertension 2002;39: 865-873.

59 Jiang L, et al: Calpain-1 regulation of matrix metalloproteinase 2 activity in vascular smooth muscle cells facilitates age-associated aortic wall calcification and fibrosis. Hypertension 2012;60:1192-1199.

60 Nabeshima Y, et al: Calpain 1 inhibitor BDA410 ameliorates $\alpha$-klotho-deficiency phenotypes resembling human aging-related syndromes. Sci Rep 2014;4:5847.

61 Davies HA, et al: Oxidative stress alters the morphology and toxicity of aortic medial amyloid. Biophys J 2015;109:2363-2370.

62 Häggqvist B, et al: Medin: an integral fragment of aortic smooth muscle cell-produced lactadherin forms the most common human amyloid. Proc Natl Acad Sci USA 1999;96: 8669-8674.

63 Peng S, et al: Medin and medin-amyloid in ageing inflamed and non-inflamed temporal arteries. J Pathol 2002;196:91-96.

64 Peng S, Glennert J, Westermark P: Medinamyloid: a recently characterized age-associated arterial amyloid form affects mainly arteries in the upper part of the body. Amyloid 2005; $12: 96-102$.

65 Larsson A, et al: Lactadherin binds to elastin - a starting point for medin amyloid formation? Amyloid 2006;13:78-85.

66 Brissette MJ, et al: MFG-E8 released by apoptotic endothelial cells triggers anti-inflammatory macrophage reprogramming. PLoS One 2012; 7:e36368.

67 Thorp E, Tabas I: Mechanisms and consequences of efferocytosis in advanced atherosclerosis. J Leukoc Biol 2009;86:1089-1095.

68 Ait-Oufella H, et al: Lactadherin deficiency leads to apoptotic cell accumulation and accelerated atherosclerosis in mice. Circulation 2007;115:2168-2177.

69 Fleenor BS, et al: Curcumin ameliorates arterial dysfunction and oxidative stress with aging. Exp Gerontol 2013;48:269-276.

70 Gong X, et al: Long-term atorvastatin improves age-related endothelial dysfunction by ameliorating oxidative stress and normalizing eNOS/iNOS imbalance in rat aorta. Exp Gerontol 2014;52C:9-17.

71 Roos CM, et al: Transcriptional and phenotypic changes in aorta and aortic valve with aging and MnSOD deficiency in mice. Am J Physiol Heart Circ Physiol 2013;305:H1428H1439.

72 Lund DD, et al: Protective effect of extracellular superoxide dismutase on endothelial function during aging. Am J Physiol Heart Circ Physiol 2009;296:H1920-H1925.

73 Rice KM, et al: Chronic paracetamol treatment influences indices of reactive oxygen species accumulation in the aging Fischer 344 $\mathrm{X}$ Brown Norway rat aorta. Ann Clin Lab Sci 2012;42:152-161

74 van der Loo B, et al: Enhanced peroxynitrite formation is associated with vascular aging. J Exp Med 2000;192:1731-1744.

75 Wang M, et al: Involvement of NADPH oxidase in age-associated cardiac remodeling. J Mol Cell Cardiol 2010;48:765-772.

76 Barrett-O'Keefe Z, et al: Endothelin-A-mediated vasoconstriction during exercise with advancing age. J Gerontol A Biol Sci Med Sci 2015;70:554-565.

77 Wirth A, et al: Age-dependent blood pressure elevation is due to increased vascular smooth muscle tone mediated by G-protein signalling. Cardiovasc Res 2016;109:131-140.

78 Fleenor BS, et al: Arterial stiffening with ageing is associated with transforming growth factor- $\beta 1$-related changes in adventitial collagen: reversal by aerobic exercise. J Physiol 2010;588:3971-3982.

79 Vendrov AE, et al: NOX4 NADPH oxidasedependent mitochondrial oxidative stress in aging-associated cardiovascular disease. Antioxid Redox Signal 2015;23:1389-1409.

80 Mikhed Y, Daiber A, Steven S: Mitochondrial oxidative stress, mitochondrial DNA damage and their role in age-related vascular dysfunction. Int J Mol Sci 2015;16:15918-15953.

$81 \mathrm{Kim}$ MY, et al: The PPAR $\delta$-mediated inhibition of angiotensin II-induced premature senescence in human endothelial cells is SIRT1dependent. Biochem Pharmacol 2012;84: 1627-1634.

82 Craige SE, Keaney JF Jr: Polyploidy and dysregulated ROS signaling: the school of hard Nox? Cell Cycle 2009;8:797.

83 Zarzuelo MJ, et al: SIRT1 inhibits NADPH oxidase activation and protects endothelial function in the rat aorta: implications for vascular aging. Biochem Pharmacol 2013;85: 1288-1296.

84 Santhanam L, et al: Decreased S-nitrosylation of tissue transglutaminase contributes to agerelated increases in vascular stiffness. Circ Res 2010;107:117-125.

85 Roos CM, et al: Chronic senolytic treatment alleviates established vasomotor dysfunction in aged or atherosclerotic mice. Aging Cell 2016;15:973-977.

86 Donato AJ, et al: Life-long caloric restriction reduces oxidative stress and preserves nitric oxide bioavailability and function in arteries of old mice. Aging Cell 2013;12: 772-783.

87 Donato AJ, et al: SIRT-1 and vascular endothelial dysfunction with ageing in mice and humans. J Physiol 2011;589:4545-4554.

88 Novella S, et al: Aging-related endothelial dysfunction in the aorta from female senescence-accelerated mice is associated with decreased nitric oxide synthase expression. Exp Gerontol 2013;48:1329-1337.

89 Steppan J, et al: Exercise, vascular stiffness, and tissue transglutaminase. J Am Heart Assoc 2014;3:e000599. 
90 Hezel M, et al: Dietary nitrate improves agerelated hypertension and metabolic abnormalities in rats via modulation of angiotensin II receptor signaling and inhibition of superoxide generation. Free Radic Biol Med 2016;99:87-98.

91 Alghasham A, et al: Impact of anti-peroxynitrite-damaged-thymidine-monophosphate antibodies on disease activity in patients with systemic lupus erythematosus. Nucleosides Nucleotides Nucleic Acids 2015;34: 56-68.

92 Csiszar A, et al: Age-associated proinflammatory secretory phenotype in vascular smooth muscle cells from the non-human primate Macaca mulatta: reversal by resveratrol treatment. J Gerontol A Biol Sci Med Sci 2012;67:811-820.

93 Donato AJ, et al: Direct evidence of endothelial oxidative stress with aging in humans: relation to impaired endothelium-dependent dilation and upregulation of nuclear factor- $\kappa$ B. Circ Res 2007;100:1659-1666.

94 Donato AJ, et al: Aging is associated with greater nuclear $\mathrm{NF} \kappa \mathrm{B}$, reduced $\mathrm{I} \kappa \mathrm{B} \alpha$, and increased expression of proinflammatory cytokines in vascular endothelial cells of healthy humans. Aging Cell 2008;7:805812.

95 Ungvari Z, et al: Age-associated vascular oxidative stress, Nrf2 dysfunction, and NF- $\mathrm{kB}$ activation in the nonhuman primate $\mathrm{Macaca}$ mulatta. J Gerontol A Biol Sci Med Sci 2011; 66:866-875.

96 Tang Y, et al: Resveratrol reduces vascular cell senescence through attenuation of oxidative stress by SIRT1/NADPH oxidase-dependent mechanisms. J Nutr Biochem 2012; 23:1410-1416.

97 Thompson AM, Wagner R, Rzucidlo EM: Age-related loss of SirT1 expression results in dysregulated human vascular smooth muscle cell function. Am J Physiol Heart Circ Physiol 2014;307:H533-H541.

98 Badi I, et al: MicroRNA-34a induces vascular smooth muscle cells senescence by SIRT1 downregulation and promotes the expression of age-associated pro-inflammatory secretory factors. J Gerontol A Biol Sci Med Sci 2015;70:1304-1311.

99 Bochenek ML, Schütz E, Schäfer K: Endothelial cell senescence and thrombosis: ageing clots. Thromb Res 2016;147:36-45.

100 Herbert KE, et al: Angiotensin II-mediated oxidative DNA damage accelerates cellular senescence in cultured human vascular smooth muscle cells via telomere-dependent and independent pathways. Circ Res 2008; 102:201-208.

101 Shan H, et al: From autophagy to senescence and apoptosis in Angiotensin II-treated vascular endothelial cells. APMIS 2014;122: 985-992.

102 Shan H, Bai X, Chen X: Angiotensin II induces endothelial cell senescence via the activation of mitogen-activated protein kinases. Cell Biochem Funct 2008;26:459-466.
103 Tanaka Y, Moritoh Y, Miwa N: Age-dependent telomere-shortening is repressed by phosphorylated $\alpha$-tocopherol together with cellular longevity and intracellular oxidative-stress reduction in human brain microvascular endotheliocytes. J Cell Biochem 2007;102:689-703.

104 Chang E, Harley CB: Telomere length and replicative aging in human vascular tissues. Proc Natl Acad Sci USA 1995;92:1119011194.

105 Beyer AM, et al: Critical role for telomerase in the mechanism of flow-mediated dilation in the human microcirculation. Circ Res 2016;118:856-866.

106 Gardner SE, et al: Senescent vascular smooth muscle cells drive inflammation through an interleukin-1 $\alpha$-dependent senescence-associated secretory phenotype. Arterioscler Thromb Vasc Biol 2015;35:1963-1974.

107 Kinoshita D, et al: Progerin impairs vascular smooth muscle cell growth via the DNA damage response pathway. Oncotarget 2017;8:34045-34056.

108 Uryga AK, Bennett MR: Ageing induced vascular smooth muscle cell senescence in atherosclerosis. J Physiol 2016;594:21152124.

109 Wang J, et al: Vascular smooth muscle cell senescence promotes atherosclerosis and features of plaque vulnerability. Circulation 2015;132:1909-1919.

110 Watson A, et al: Nicotinamide phosphoribosyltransferase in smooth muscle cells maintains genome integrity, resists aortic medial degeneration, and is suppressed in human thoracic aortic aneurysm disease. Circ Res 2017;120:1889-1902.

111 McCrann DJ, et al: Upregulation of Nox4 in the aging vasculature and its association with smooth muscle cell polyploidy. Cell Cycle 2009;8:902-908.

112 Mistry Y, et al: A role for mitochondrial oxidants in stress-induced premature senescence of human vascular smooth muscle cells. Redox Biol 2013;1:411-417.

113 Porter LJ, et al: Prelamin A accumulation attenuates Racl activity and increases the intrinsic migrational persistence of aged vascular smooth muscle cells. Cells 2016; 5:E41.

114 Bennett MR, Sinha S, Owens GK: Vascular smooth muscle cells in atherosclerosis. Circ Res 2016;118:692-702.

115 Wang M, et al: MFG-E8 activates proliferation of vascular smooth muscle cells via integrin signaling. Aging Cell 2012;11:500508.

116 Lakatta EG, Wang M, Najjar SS: Arterial aging and subclinical arterial disease are fundamentally intertwined at macroscopic and molecular levels. Med Clin North Am 2009; 93:583-604.

117 Humphrey JD, Dufresne ER, Schwartz MA: Mechanotransduction and extracellular matrix homeostasis. Nat Rev Mol Cell Biol 2014;15:802-812.
118 Sehgel NL, Vatner SF, Meininger GA: "Smooth muscle cell stiffness syndrome" revisiting the structural basis of arterial stiffness. Front Physiol 2015;6:335.

119 Qiu H, et al: Short communication: vascular smooth muscle cell stiffness as a mechanism for increased aortic stiffness with aging. Circ Res 2010;107:615-619.

120 Langlois B, et al: Vimentin knockout results in increased expression of sub-endothelial basement membrane components and carotid stiffness in mice. Sci Rep 2017;7:11628.

121 Galmiche G, et al: Inactivation of serum response factor contributes to decrease vascular muscular tone and arterial stiffness in mice. Circ Res 2013;112:1035-1045.

$122 \mathrm{Zhu}$ W, et al: A new twist to the primitive mechanical fate identifies an aged-associated TGF $\beta 1$ nexus in vascular smooth muscle cells. FASEB J 2016;30(suppl 1):abstract 1300.3.

123 Steppan J, et al: Tissue transglutaminase modulates vascular stiffness and function through crosslinking-dependent and crosslinking-independent functions. J Am Heart Assoc 2017;6:e004161.

124 Dinardo CL, et al: Variation of mechanical properties and quantitative proteomics of VSMC along the arterial tree. Am J Physiol Heart Circ Physiol 2014;306:H505-H516.

125 Sehgel NL, et al: Augmented vascular smooth muscle cell stiffness and adhesion when hypertension is superimposed on aging. Hypertension 2015;65:370-377.

126 Chatterjee S, et al: Mechanosignaling in the vasculature: emerging concepts in sensing, transduction and physiological responses. Am J Physiol Heart Circ Physiol 2015; 308:H1451-H1462.

127 Burton DG, Matsubara H, Ikeda K: Pathophysiology of vascular calcification: pivotal role of cellular senescence in vascular smooth muscle cells. Exp Gerontol 2010;45: 819-824.

128 Cattell MA, Anderson JC, Hasleton PS: Agerelated changes in amounts and concentrations of collagen and elastin in normotensive human thoracic aorta. Clin Chim Acta 1996; 245:73-84.

129 Taghizadeh H, Tafazzoli-Shadpour M: Characterization of mechanical properties of lamellar structure of the aortic wall: effect of aging. J Mech Behav Biomed Mater 2016; 65:20-28.

130 Antonicelli F, et al: Elastin-elastases and inflamm-aging. Curr Top Dev Biol 2007;79: 99-155.

131 Maurice P, et al: Elastin fragmentation and atherosclerosis progression: the elastokine concept. Trends Cardiovasc Med 2013;23: 211-221.

132 Duca L, et al: Matrix ageing and vascular impacts: focus on elastin fragmentation. Cardiovasc Res 2016;110:298-308.

133 Fritze O, et al: Age-related changes in the elastic tissue of the human aorta. J Vasc Res 2012;49:77-86. 
134 Ferruzzi J, et al: Loss of elastic fiber integrity compromises common carotid artery function: implications for vascular aging. Artery Res 2016;14:41-52.

135 Joly L, et al: Influence of thoracic aortic inflammation and calcifications on arterial stiffness and cardiac function in older subjects. J Nutr Health Aging 2016;20:347-354.

136 Derlin T, et al: Age-related differences in the activity of arterial mineral deposition and regional bone metabolism: ${ }^{18} \mathrm{~F}$-sodium fluoride positron emission tomography study. Osteoporos Int 2015;26:199-207.

137 Lee SH, et al: Coronary calcification is reversely related with bone and hair calcium: the relationship among different calcium pools in body. J Bone Metab 2016;23:191-197.

138 Ouyang L, et al: Roles of platelet-derived growth factor in vascular calcification. J Cell Physiol 2018;233:2804-2814.

139 Semba RD, et al: Serum carboxymethyllysine, an advanced glycation end product, is associated with arterial stiffness in older adults. J Hypertens 2015;33:797-803; discussion 803

140 Wang Y, et al: Effect of glucose on the biomechanical function of arterial elastin. J Mech Behav Biomed Mater 2015;49:244254.

141 Lesniewski LA, et al: Aerobic exercise reverses arterial inflammation with aging in mice. Am J Physiol Heart Circ Physiol 2011; 301:H1025-H1032.

$142 \mathrm{Al}$ Mheid I, et al: Age and human regenerative capacity impact of cardiovascular risk factors. Circ Res 2016;119:801-809.

143 AlGhatrif M, et al: Longitudinal trajectories of arterial stiffness and the role of blood pressure: the Baltimore Longitudinal Study of Aging. Hypertension 2013;62:934-941.

144 Fortier C, et al: Aortic-brachial pulse wave velocity ratio: a blood pressure-independent index of vascular aging. Hypertension 2017; 69:96-101.

145 Furman D, et al: Expression of specific inflammasome gene modules stratifies older individuals into two extreme clinical and immunological states. Nat Med 2017;23: 174-184.

146 Humphrey JD, Milewicz DM: Aging, smooth muscle vitality, and aortic integrity. Circ Res 2017;120:1849-1851.

147 Mitchell GF, et al: Arterial stiffness, pressure and flow pulsatility and brain structure and function: the Age, Gene/Environment Susceptibility - Reykjavik study. Brain 2011; 134(pt 11):3398-3407.

148 Paneni F, et al: The aging cardiovascular system: understanding it at the cellular and clinical levels. J Am Coll Cardiol 2017;69: 1952-1967.

149 Whitlock MC, Hundley WG: Noninvasive imaging of flow and vascular function in disease of the aorta. JACC Cardiovasc Imaging 2015;8:1094-1106.

150 Virmani R, et al: Effect of aging on aortic morphology in populations with high and low prevalence of hypertension and atherosclerosis. Comparison between occidental and Chinese communities. Am J Pathol 1991;139:1119-1129.

151 Niiranen TJ, et al: Aortic-brachial arterial stiffness gradient and cardiovascular risk in the community: the Framingham Heart Study. Hypertension 2017;69:1022-1028.

152 Raghu R, et al: Comparative study of viscoelastic arterial wall models in nonlinear onedimensional finite element simulations of blood flow. J Biomech Eng 2011;133:081003.

153 Majesky MW, et al: Vascular smooth muscle progenitor cells: building and repairing blood vessels. Circ Res 2011;108:365-377.

154 Yin H, Pickering JG: Cellular senescence and vascular disease: novel routes to better understanding and therapy. Can J Cardiol 2016;32:612-623.

155 Yeh HI, et al: Age-related alteration of gap junction distribution and connexin expression in rat aortic endothelium. J Histochem Cytochem 2000;48:1377-1389.

$156 \mathrm{Du} \mathrm{W}$, et al: Age-associated vascular inflammation promotes monocytosis during atherogenesis. Aging Cell 2016;15:766-777.

157 Guzik TJ, Cosentino F: Epigenetics and immunometabolism in diabetes and aging. Antioxid Redox Signal 2018;29:257-274.

158 Nogueira-Neto J, et al: Basal neutrophil function in human aging: implications in endothelial cell adhesion. Cell Biol Int 2016; 40:796-802.

159 Joffre J, et al: Genetic and pharmacological inhibition of TREM-1 limits the development of experimental atherosclerosis. J Am Coll Cardiol 2016;68:2776-2793.

160 Niiranen TJ, et al: Prevalence, correlates, and prognosis of healthy vascular aging in a Western community-dwelling cohort: the Framingham Heart Study. Hypertension 2017;70:267-274.

161 Sazonova OV, et al: Extracellular matrix presentation modulates vascular smooth muscle cell mechanotransduction. Matrix Biol 2015;41:36-43.

162 Tan I, et al: Effect of heart rate on arterial stiffness as assessed by pulse wave velocity. Curr Hypertens Rev 2017, Epub ahead of print.

$163 \mathrm{Xiao} \mathrm{H}$, et al: Arterial viscoelasticity: role in the dependency of pulse wave velocity on heart rate in conduit arteries. Am J Physiol Heart Circ Physiol 2017;312:H1185H1194.

164 Mattison JA, et al: Resveratrol prevents high fat/sucrose diet-induced central arterial wall inflammation and stiffening in nonhuman primates. Cell Metab 2014;20:183-190.

165 Najjar SS, et al: Pulse wave velocity is an independent predictor of the longitudinal increase in systolic blood pressure and of incident hypertension in the Baltimore Longitudinal Study of Aging. J Am Coll Cardiol 2008;51:1377-1383.

166 Franklin SS, et al: Hemodynamic patterns of age-related changes in blood pressure. The
Framingham Heart Study. Circulation 1997; 96:308-315.

167 Malavolta M, et al: Pleiotropic effects of tocotrienols and quercetin on cellular senescence: introducing the perspective of senolytic effects of phytochemicals. Curr Drug Targets 2016;17:447-459.

168 Janubová M, Žitňanová I: Effects of bioactive compounds on senescence and components of senescence associated secretory phenotypes in vitro. Food Funct 2017;8 2394-2418.

169 Schmitt R: Senotherapy: growing old and staying young? Pflugers Arch 2017;469: 1051-1059.

170 Mattison JA, et al: Caloric restriction improves health and survival of rhesus monkeys. Nat Commun 2017;8:14063.

171 Mattison JA, et al: Impact of caloric restriction on health and survival in rhesus monkeys from the NIA study. Nature 2012;489: 318-321.

172 Weiss EP, Fontana L: Caloric restriction: powerful protection for the aging heart and vasculature. Am J Physiol Heart Circ Physiol 2011;301:H1205-H1219.

173 Rossman MJ, et al: Endothelial cell senescence with aging in healthy humans: prevention by habitual exercise and relation to vascular endothelial function. Am J Physiol Heart Circ Physiol 2017;313:H890-H895.

174 Gauche R, et al: Blood pressure reactivity to mental stress is attenuated following resistance exercise in older hypertensive women. Clin Interv Aging 2017;12:793-803.

175 Zhu Y, et al: The Achilles' heel of senescent cells: from transcriptome to senolytic drugs. Aging Cell 2015;14:644-658.

176 Childs BG, et al: Senescent intimal foam cells are deleterious at all stages of atherosclerosis. Science 2016;354:472-477.

177 Jeon $\mathrm{OH}$, et al: Local clearance of senescent cells attenuates the development of posttraumatic osteoarthritis and creates a proregenerative environment. Nat Med 2017; 23:775-781.

178 Huang W, Alhenc Gelas F, Osborne-Pellegrin MJ: Protection of the arterial internal elastic lamina by inhibition of the reninangiotensin system in the rat. Circ Res 1998; 82:879-890

179 Wang M, et al: Method for the diagnosis of age-associated vascular disorders. WO/ 2010/096713. 2010.

180 Baur JA, et al: Resveratrol improves health and survival of mice on a high-calorie diet. Nature 2006;444:337-342.

181 Lesniewski LA, et al: Dietary rapamycin supplementation reverses age-related vascular dysfunction and oxidative stress, while modulating nutrient-sensing, cell cycle, and senescence pathways. Aging Cell 2017;16:1726.

$182 \mathrm{Xu}$ X, et al: Metformin protects against systolic overload-induced heart failure independent of AMP-activated protein kinase a2. Hypertension 2014;63:723-728. 